\title{
Reverse Shock Emission and Ionization Break Out Powered by Post-merger Millisecond Magnetars
}

\author{
Ling-Jun Wang ${ }^{1,2}$, Zi-Gao Dai ${ }^{1,2}$, and Yun-Wei Yu ${ }^{3}$ \\ ${ }^{1}$ School of Astronomy and Space Science, Nanjing University, Nanjing, China; \\ dzg@nju.edu.cn \\ ${ }^{2}$ Key laboratory of Modern Astronomy and Astrophysics (Nanjing University), Ministry of \\ Education, Nanjing 210093, China \\ ${ }^{3}$ Institute of Astrophysics, Central China Normal University, Wuhan 430079, China; \\ yuyw@mail.ccnu.edu.cn
}

\begin{abstract}
There is accumulating evidence that at least a fraction of binary neutron star mergers result in rapidly spinning magnetars, with subrelativistic neutron-rich ejecta as massive as a small fraction of solar mass. The ejecta could be heated continuously by the Poynting flux emanated from the central magnetars. Such Poynting flux could become lepton-dominated so that a reverse shock develops. It was demonstrated that such a picture is capable of accounting for the optical transient PTF11agg (Wang \& Dai 2013b). In this paper we investigate the Xray and ultraviolet (UV) radiation as well as the optical and radio radiation studied by Wang \& Dai (2013b). UV emission is particularly important because it has the right energy to ionize the hot ejecta at times $t \lesssim 600$ s. It is thought that the ejecta of binary neutron star mergers are a remarkably pure sample of $r$-process material, about which our understanding is still incomplete. In this paper we evaluate the possibility of observationally determining the bound-bound and bound-free opacities of the $r$-process material by timing the X-ray, UV, and optical radiation. It is found that these timings depend on the opacities weakly and therefore only loose constraints on the opacities can be obtained.
\end{abstract}

Subject headings: radiation mechanisms: non-thermal — stars: neutron

\section{Introduction}

Compact binary mergers are of great astrophysical importance as the primary sources for the upcoming next generation ground-based gravitational wave (GW) detectors (see 
Abadie et al. 2010; Bartos et al. 2013, for recent reviews). The detection of GW can be confirmed if accompanying electromagnetic (EM) signals are detected at the same time.

It has been believed that the mergers of compact binaries, i.e., double neutron stars (NSs) or a NS with a stellar-mass black hole, are the progenitors of short gamma-ray bursts (SGRBs; Paczyński 1986; Eichler et al. 1989; Barthelmy et al. 2005; Fox et al. 2005; Gehrels et al. 2005; Rezzolla et al. 2011). The evidence for such a scenario has recently gained strong support when Berger et al. (2013) and Tanvir et al. (2013) discovered an rprocess kilonova associated with the SGRB 130603B ${ }^{1}$. Kilonova, powered radioactively by the neutron-rich material ejected during the coalescence of compact binaries, was first analytically predicted by Li \& Paczyński (1998) and then intensely studied by many authors (Kulkarni 2005; Rosswog 2005; Metzger et al. 2010b; Roberts et al. 2011; Metzger \& Berger 2012). Other EM signals include radio afterglows (Nakar \& Piran 2011; Metzger \& Berger 2012; Piran et al. 2013; Rosswog et al. 2013) and possible X-ray emission produced by the interaction of the NS magnetospheres during the inspiral and merger process (Palenzuela et al. 2013).

In a popular scenario, the hypermassive remnant of binary neutron star (BNS) merger collapses into a black hole on a timescale of $\sim 200 \mathrm{~ms}$ (Faber \& Rasio 2012). Models involving a black hole surrounded by a hyperaccretion disc have been proposed (Popham et al. 1999; Narayan et al. 2001; Kohri \& Mineshige 2002; Xue et al. 2013; Kawanaka et al. 2013) as the central engine of GRBs. Several authors (Dai et al. 2006; Zhang 2013), however, suggest that a highly-magnetized, rapidly spinning stable NS (magnetar) could form during the coalescence of BNSs. The evidence for magnetar formation following some SGRBs is accumulated during the past decade (Dai et al. 2006; Fan \& Xu 2006; Gao \& Fan 2006; Rowlinson et al. 2010, 2013; Dai \& Liu 2012; Wang \& Dai 2013b; Gompertz et al. 2014). In addition, magnetar activity could also be responsible for the statistical properties of X-ray flares from some GRBs (Wang \& Dai 2013a). Recent simulations in numerical relativity (e.g. Giacomazzo \& Perna 2013) confirmed that formation of a stable long-lived magnetar is a plausible scenario. However, in some cases the presence of such stable long-lived magnetars has been ruled out by radio observations, as in the cases of GRB 050724 and GRB 060505 (Metzger \& Bower 2014).

Zhang (2013) recently proposed that if hypermassive millisecond magnetar resulting from a coalescence of BNSs survives for sufficiently long time, a significant fraction of NS-NS

\footnotetext{
${ }^{1}$ Jin et al. (2013) put forward an alternative scenario based on a two-component jet model. The nondetection of late radio emission of GRB 130603B (Fong et al. 2014) does not favor such an interpretation though. Despite of this fact, other possibilities (Fan et al. 2013; Takami et al. 2014) cannot be ruled out on current observational ground.
} 
mergers would be discovered as bright X-ray transients associated with GW bursts without SGRB association. Gao et al. (2013) further studied the broadband EM signals of forward shock (FS) driven by the ejecta based on the energy injection scenario (Dai \& Lu 1998a,b; Zhang \& Mészáros 2001). Subsequently, Yu et al. (2013) studied the supernova-like EM signals of ejecta (dubbed merger-nova) powered by the Poynting flux from post-merger magnetars by assuming the complete absorption of Poynting flux by ejecta. Wang \& Dai (2013b), on the other hand, analytically studied the reverse shock (RS) emission powered by the central millisecond magnetars based on the fact that the Poynting flux is more likely to become lepton-dominated ( $e^{+} e^{-}$pairs; Coroniti 1990; Michel 1994; Dai 2004; Yu \& Dai 2007). Such a scenario successfully interprets observational data of the optical transient PTF11agg discovered by the Palomar Transient Factory group (Cenko et al. 2013; Wang \& Dai 2013b) ${ }^{2}$. More recently Metzger \& Piro (2014) investigated the effects of pair-creation and annihilation on the optical and X-ray emission from the remnant of BNSs mergers powered by stable millisecond magnetars.

One of the major concerns in the theory of BNS mergers is the uncertainty in the optical and nuclear properties of the ejected matter, which have started to attract attention only recently (Goriely et al. 2011; Korobkin et al. 2012; Barnes \& Kasen 2013; Bauswein et al. 2013; Grossman et al. 2014; Tanaka \& Hotokezaka 2013). In particular, the opacity of the ejecta was usually set as $\kappa=0.2 \mathrm{~cm}^{2} \mathrm{~g}^{-1}$ in the previous studies (e.g., Li \& Paczyński 1998; Rosswog 2005; Roberts et al. 2011; Yu et al. 2013), which was recently found to be $\kappa \sim$ $10 \mathrm{~cm}^{2} \mathrm{~g}^{-1}$ (Barnes \& Kasen 2013; Kasen et al. 2013; Tanaka \& Hotokezaka 2013; Grossman et al. 2014). As a result of much higher values of opacity, much redder and dimmer kilonova transients are expected, with their peak shifted to infrared, just as in the case of event associated with SGRB 130603B (Berger et al. 2013; Tanvir et al. 2013).

The ejecta from a neutron star merger (NSM) are thought to be a pure sample of $r$ process material. Apart from the above theoretical studies of the $r$-process material, it will be particularly valuable to observationally constrain the opacity of the $r$-process material. In this paper, we evaluate the possibility to constrain the bound-free and bound-bound opacities, which are strongly dependent on the nuclear composition of the ejecta. The physical picture of this scenario is illustrated in Figure 1. High opacity of the ejecta is caused by the presence of elements with half-filled $f$-shells, such as lanthanides and actinides produced in the $r$-process (Kasen et al. 2013). As a result of high (bound-bound) opacity, optical emission from RS would be blocked during the early expansion of the ejecta. Early X-ray emission from the RS, on the other hand, would be blocked only by the electron

\footnotetext{
${ }^{2} \mathrm{Wu}$ et al. (2014) proposed an alternative interpretation for this optical transient within the framework of a magnetar-powered blast wave.
} 
scattering opacity and unaffected by the bound-bound transitions. Consequently, we can determine the launch time of the Poynting flux more accurately, more definitively by timing $\mathrm{X}$-ray emission. UV radiation from RS, however, is of great importance because it has the right energy to ionize the hot ejecta. Through a fortunate observation of X-ray, UV, and optical emission from RS powered by a millisecond magnetar, we can determine the launch time of the central magnetar (by X-ray observation), ionization break out by the UV radiation and the time when the ejecta become optically transparent. The later two times depend on the bound-free and bound-bound opacity, respectively.

In Wang \& Dai (2013b), we demonstrated how our model consistently accounts for the observed optical and radio properties of the PTF11agg transient. We considered the case in which the spin-down time of the magnetar $T_{\text {sd }}$ exceeds the deceleration time of the blast wave $T_{\text {dec }}$ (e.g., Case I of Gao et al. 2013). In Section 2, we further study the other two cases. In our calculations, we adopt an improved version of the blast wave dynamics, which is different from the one used in Gao et al. (2013) and Wang \& Dai (2013b) in that it accounts for the different regions in the blast wave. We compare different prescriptions for the dynamics in Section 2. In the same section we additionally investigate the effect of the optical obscuration by the $r$-process material, neglected in Wang \& Dai (2013b). We study the ionization effect of the UV radiation on the ejecta in Section 3. Finally, we discuss how to observationally constrain the bound-bound and bound-free opacities in Section 4. A summary is given in Section 5.

\section{Leptonized reverse shock emission}

Numerical simulations suggested that the ejecta from an NSM have a typical velocity $v=0.1-0.3 c$ and mass $M_{\mathrm{ej}}=10^{-4}-10^{-2} M_{\odot}$ (Rezzolla et al. 2010; Hotokezaka et al. 2013; Rosswog et al. 2013). The resulting central compact object, under the assumption that it can avoid collapse and persist for a long time, would dissipate its rotational energy by launching a luminous Poynting flux. Without the impact of the Poynting flux from the nascent millisecond magnetar, the matter ejected during the merger would have spatially extended morphology, which would soon have started to expand homologously, despite the effects of radioactive heating (Rosswog et al. 2014). With the onset of Poynting flux, the ejecta can be rapidly compressed into a thin shell by the overpressure caused by the energy injection. The overpressure will lead to a shock driving into the ejecta. The postshock pressure is $p_{2}=2 \gamma \rho_{1} v_{1}^{2} /(\gamma+1)$ for strong shock (Landau \& Lifshitz 1987), where $v_{1}$ is the unshocked fluid velocity relative to the contact discontinuity, $\rho_{1}$ its density. With $\rho_{1}=$ 
$M_{\mathrm{ej}}\left(\frac{4}{3} \pi v^{3} t^{3}\right)^{-1},{ }^{3} p_{2}=L_{\mathrm{sd}} t\left[3 \times \frac{4}{3} \pi\left(v+v_{1}\right)^{3} t^{3}\right]^{-1}$ and the width of the initial ejecta $\Delta=v_{1} t$, we find the crossing time

$$
t_{\text {cross }}=\frac{\Delta}{v_{1}}=\left(\frac{6 \gamma}{\gamma+1} \frac{\Delta^{2} M_{\mathrm{ej}}}{L_{\mathrm{sd}}}\right)^{1 / 3}=0.09 \mathrm{~s} \Delta_{7}^{2 / 3} M_{\mathrm{ej},-4}^{1 / 3} L_{\mathrm{sd}, 47}^{-1 / 3}
$$

and $v_{1}$ at this time

$$
v_{1, \mathrm{cross}}=3.8 \times 10^{-3} c \Delta_{7}^{1 / 3} M_{\mathrm{ej},-4}^{-1 / 3} L_{\mathrm{sd}, 47}^{1 / 3} \ll v \approx 0.2 c,
$$

where $L_{\text {sd }}$ is the spin-down luminosity of the magnetar. Here the usual convention $Q=10^{n} Q_{n}$ is adopted. This crossing time is negligible compared to the activity duration of the central magnetars, justifying the model we are considering.

As mentioned in Section 1, the Poynting flux from millisecond magnetars is more likely to become lepton-dominated. In developing a model to account for the transient source PTF11agg, Wang \& Dai (2013b) adopted a dynamics

$$
L_{0} \min \left(t, T_{\mathrm{sd}}\right)=\left(\gamma-\gamma_{\mathrm{ej}, 0}\right) M_{\mathrm{ej}} c^{2}+2\left(\gamma^{2}-1\right) M_{\mathrm{sw}} c^{2}
$$

which is different by a factor of 2 on the second term from that used by Gao et al. (2013). In the above equation $L_{0}=\xi L_{\mathrm{sd}}$ with $\xi$ the fraction of the Poynting flux catched by the ejecta, $\gamma$ the Lorentz factor of the ejecta with initial Lorentz factor $\gamma_{\mathrm{ej}, 0}, M_{\mathrm{sw}}$ the mass swept up by the shock.

In this paper we would like to adopt an alternate dynamics similar to that used by $\mathrm{Yu}$ et al. (2013) and to evaluate any differences between these two kinds of dynamics. In this scenario, the ejecta gain energy because of the work done by RS and likewise the FS gains energy by the work done by the ejecta. As a result, the ejecta are sandwiched between RS and FS. The treatment of RS is otherwise similar to that in Wang \& Dai (2013b).

The total non-rest energy of the ejecta and shocked media (including FS and RS) can be expressed as

$$
E_{k}=\gamma E_{3}^{\prime}+\left(\gamma-\gamma_{\mathrm{ej}, 0}\right) M_{\mathrm{ej}} c^{2}+\gamma E_{\mathrm{ej}, \mathrm{int}}^{\prime}+\left(\gamma^{2}-1\right) M_{\mathrm{sw}} c^{2}
$$

where $E_{3}^{\prime}$ and $E_{\mathrm{ej} \text {,int }}^{\prime}$ are the respective energies of the reverse-shocked wind (region 3) and ejecta in the comoving frame. For the definition of regions 1-4, see Figure 1 (see also Dai 2004). Here we neglect the rest energy of region 3 because it is lepton-dominated and

\footnotetext{
${ }^{3}$ Here we ignore the initial size of the ejecta.
} 
therefore very hot. In a way similar to the generic dynamic model for GRB afterglow (Huang et al. 1999), the dynamics can be derived as (cf. Yu et al. 2013)

$$
\frac{d \gamma}{d t}=\frac{\xi L_{\mathrm{sd}}+L_{\mathrm{rd}}-L_{\mathrm{ej}, e}-\gamma \mathcal{D}\left(\frac{d E_{3}^{\prime}}{d t^{\prime}}+\frac{d E_{\mathrm{ej}, \mathrm{int}}^{\prime}}{d t^{\prime}}\right)-\left(\gamma^{2}-1\right) c^{2}\left(\frac{d M_{\mathrm{sw}}}{d t}\right)}{E_{3}^{\prime}+M_{\mathrm{ej}} c^{2}+E_{\mathrm{ej}, \mathrm{int}}^{\prime}+2 \gamma M_{\mathrm{sw}} c^{2}}
$$

where $\mathcal{D}$ is the Doppler factor, $L_{\mathrm{rd}}$ the radioactive luminosity of the ejecta in the observer frame, $L_{\mathrm{ej}, e}$ the energy loss rate in ejecta due to thermal radiation. The evolution of energies in region 3 and ejecta can be expressed as

$$
\begin{aligned}
\frac{d E_{3}^{\prime}}{d t^{\prime}} & =\xi L_{\mathrm{sd}}^{\prime}-p_{3} \frac{d V_{3, \mathrm{enc}}^{\prime}}{d t^{\prime}} \\
\frac{d E_{\mathrm{ej}, \mathrm{int}}^{\prime}}{d t^{\prime}} & =p_{3} \frac{d V_{3, \mathrm{enc}}^{\prime}}{d t^{\prime}}-p_{\mathrm{ej}} \frac{d V_{\mathrm{ej}, \mathrm{enc}}^{\prime}}{d t^{\prime}}+L_{\mathrm{rd}}^{\prime}-L_{\mathrm{ej}, e}^{\prime},
\end{aligned}
$$

where $t^{\prime}$ is the time measured in the comoving frame, $L_{\mathrm{ej}, e}^{\prime}$ the thermal radiation of ejecta. Here we have to discern different volumes involved in this problem. See Figure 1 for reference. $V_{3 \text {,enc }}^{\prime}$ and $V_{\text {ej,enc }}^{\prime}$ are the comoving volumes enclosed by region 3 and ejecta, respectively. $V_{3}^{\prime}$ and $V_{\mathrm{ej}}^{\prime}$, on the other hand, are the volumes actually occupied by these two regions. In other words, the volumes with the subscript enc denote the spherical volumes, the volumes without such subscript denote the shell volumes. The pressures in region 3 and ejecta in the comoving frame are $p_{3}=e_{3} / 3$ and $p_{\mathrm{ej}}=E_{\mathrm{ej}, \mathrm{int}}^{\prime} / 3 V_{\mathrm{ej}}^{\prime}$, respectively. The width of the sandwiched ejecta can be determined by assuming pressure balance $p_{3}=p_{\text {ej }}$. Because the ejecta are sandwiched in a thin shell, i.e., the width of ejecta (in the comoving frame) $\Delta_{\text {ej }}^{\prime} \ll r$, so that its volume $V_{\mathrm{ej}}^{\prime} \approx 0$, Equation $(7)$ can be approximately written as

$$
\frac{d E_{\mathrm{ej}, \mathrm{int}}^{\prime}}{d t^{\prime}}=L_{\mathrm{rd}}^{\prime}-L_{\mathrm{ej}, e}^{\prime}
$$

The comoving volume can be found as (Yu et al. 2013)

$$
\frac{d V_{3, \mathrm{enc}}^{\prime}}{d t^{\prime}}=4 \pi r^{2} \xi \beta c
$$

with

$$
\frac{d r}{d t}=\frac{\beta c}{1-\beta}
$$

The radioactive luminosity in the comoving frame $L_{\mathrm{rd}}^{\prime}=L_{\mathrm{rd}} / \mathcal{D}^{2}$ is determined according to Equation (4) of Korobkin et al. (2012). The thermal energy $L_{\mathrm{ej}, e}^{\prime}$ emitted by the sandwiched ejecta in the comoving frame is still expressed by Equation (7) in $\mathrm{Yu}$ et al. (2013), while the luminosity light curves at a particular observational frequency are modified as

$$
\nu L_{\nu}=\frac{1}{\max (\tau, 1)} \frac{8 \pi^{2} \mathcal{D}^{2} r^{2}}{h^{3} c^{2}} \frac{\Delta_{\mathrm{ej}}^{\prime}}{r} \frac{(h \nu / \mathcal{D})^{4}}{\exp \left(h \nu / \mathcal{D} k T^{\prime}\right)-1},
$$


i.e., there is an extra factor $\Delta_{\text {ej }}^{\prime} / r$ compared with the solid spherical geometry.

Inspection of Equation (3) indicates that initially $\left(\gamma-\gamma_{\mathrm{ej}, 0}\right) M_{\mathrm{ej}} c^{2} \gg 2\left(\gamma^{2}-1\right) M_{\mathrm{sw}} c^{2}$, the ejecta will be accelerated linearly in time until $t=\min \left(T_{\mathrm{sd}}, T_{\mathrm{dec}}\right)$, where the deceleration timescale $T_{\mathrm{dec}}$ is determined by the condition $\left(\gamma-\gamma_{\mathrm{ej}, 0}\right) M_{\mathrm{ej}} c^{2}=2\left(\gamma^{2}-1\right) M_{\mathrm{sw}} c^{2}$ (see also Gao et al. 2013). By setting the spin-down timescale $T_{\mathrm{sd}} \sim T_{\mathrm{dec}}$, we will arrive at a critical ejecta mass (Gao et al. 2013)

$$
M_{\mathrm{ej}, c} \sim 10^{-3} M_{\odot} n^{1 / 8} I_{45}^{5 / 4} L_{0,47}^{-3 / 8} P_{0,-3}^{-5 / 2} \xi^{5 / 4}
$$

where $I$ is the moment of inertia of the neutron star. It is this critical mass that defines the three cases considered by Gao et al. (2013), i.e., Case I for $T_{\mathrm{sd}}>T_{\mathrm{dec}}$, Case II for $T_{\mathrm{sd}}=T_{\mathrm{dec}}$, and Case III for $T_{\mathrm{sd}}<T_{\mathrm{dec}}$. Here we show the corresponding results including RS in Figures $2-4$, where the spectral index of electrons in FS is set as $p^{F S}=2.3$, and the luminosity distance of the source is set as $D_{L}=10^{27} \mathrm{~cm}$. The optical opacity is set as $\kappa=10 \mathrm{~cm}^{2} \mathrm{~g}^{-1}$. For more discussions about the dependence of opacity on wavelength, see Section 3. To ease comparison between different choices of dynamics, we selectively show in Figures $2 \mathrm{a}$ and $2 \mathrm{~b}$ as dashed lines the results with the same parameters as the solid lines but with dynamics expressed by Equation (3).

In the above calculations we set the Lorentz factor of the unshocked electrons/positrons (region 4) as $\gamma_{4}=10^{4}$, as determined in the literature (Atoyan 1999; Dai 2004; Wang \& Dai 2013b). The spectral index of $e^{+} e^{-}$in RS is set as $p^{R S}=2.2$ (see, e.g., Wang \& Dai 2013b). There is an additional complication concerning RS that should be mentioned. After the shut-off of the central magnetar at $T_{\text {sd }}$, the reverse shock crosses region 4 so that there are no more $e^{+} e^{-}$to be shocked. Consequently, the electrons already cooled to a Lorentz factor $\gamma_{c}$ cannot be shocked again to a higher Lorentz factor. As a result, in the analytical and numerical calculations, if we find the cooling Lorentz factor increases in a time period after $T_{\text {sd }}$, which is the case when $t>T_{N 2}$ for Case I, we will set its value the same as before.

We find that the characteristic frequencies of synchrotron radiation of FS are quite similar to that calculated by Gao et al. (2013) so that we do not show them in the figures. Because the RS emission will be absorbed by the ejecta, we show the RS emission with/without absorption in Panels (d) and (c) respectively. It is in principle possible that a FS and a RS develop in the ejecta upon the interaction with region 3 and region 2 respectively. In practice, however, since the ejecta are very thin, the FS and RS in it can last only for a transient while. Consequently we do not consider them here.

A first glimpse of Figure 2a shows that the evolution of Lorentz factor with different choices of dynamics are very similar. However, there are also differences that result in appreciable modification to the evolution of characteristic frequencies (comparing the solid 
lines and dashed lines in Figure 2b) and light curves, which are not shown in Figure 2. The reason is that the ejecta absorb some amount of energy, resulting in a heavier ejecta and therefore a delay of the deceleration time $T_{\text {dec }}$ by a factor of 2.1 . This further results in a drop of $\nu_{m}$ (see Figure 2b), which is otherwise identical to the situation where the ejecta do not absorb energy (comparing the solid lines and dashed lines in Figure 2b). The delay of $T_{\text {dec }}$ also strengthens the FS since more ambient media are swept up at $T_{\text {dec }}$. Figure $4 a$ shows the coasting of Lorentz factor, but not so clear-cut as in Figure 4a of Gao et al. (2013).

Although the energy absorption by ejecta has an appreciable effect on the dynamics and therefore the light curves, it is not so significant as for the case of merger-nova discussed by Yu et al. (2013). We therefore conclude that a simple model based on Equation (3) and used in Wang \& Dai (2013b), properly reproduces qualitative features of the more sophisticated one employed here, and therefore the use of this model in Wang \& Dai (2013b) is justified.

For completeness and also for the ease of future quantitative analysis, we present the analytical results of RS based on the dynamics (3) for Case II and Case III below and their temporal scaling indices in Table 1 (the corresponding results for Case I can be found in Wang \& Dai 2013b). For Case II, the various time scales and the peak Lorentz factor are

$$
\begin{aligned}
T_{\mathrm{N} 1}= & 2.1 \times 10^{-4} \text { days } L_{0,49}^{-1} M_{\mathrm{ej},-4} \\
T_{\mathrm{ct}}= & 2.8 \times 10^{-3} \text { days } L_{0,49}^{-2 / 3} M_{\mathrm{ej},-4}^{5 / 6} \epsilon_{B,-1}^{1 / 6} \\
T_{a c} \approx & T_{m c}=5.0 \times 10^{-3} \text { days } L_{0,49}^{-5 / 7} M_{\mathrm{ej},-4}^{6 / 7} \epsilon_{B,-1}^{1 / 7} \epsilon_{e}^{1 / 7} \gamma_{4,4}^{1 / 7} \\
T_{\mathrm{dec}} \simeq & T_{\mathrm{sd}} \\
T_{\mathrm{N} 2}= & 2.4 \times 10^{2} \text { days } L_{0,49}^{1 / 3} T_{\mathrm{sd}, 3}^{1 / 3} n^{-1 / 3} \\
T_{a m 2}= & 9.4 \times 10^{2} \text { days } L_{0,49}^{49 / 2} T_{\mathrm{sd}, 3}^{4 / 2} M_{\mathrm{ej},-4}^{-45 / 2} \gamma_{4,4}^{-30} n^{1 / 2} \epsilon_{B,-1}^{-5 / 2} \epsilon_{e}^{-25} \\
& \times\left[(p-1) \Gamma\left(\frac{3 p+22}{12}\right) \Gamma\left(\frac{3 p+2}{12}\right)\right]^{5}\left(\frac{p-1}{p-2}\right)^{25} \\
\gamma_{\mathrm{sd}}= & 28 L_{0,49} T_{\mathrm{sd}, 3} M_{\mathrm{ej},-4}^{-1}+1,
\end{aligned}
$$

where $T_{a m 2}$ is the time when $\nu_{a}$ crosses $\nu_{m}$ at the second time, which is very sensitive to many parameters because these two frequencies almost have the same temporal evolution indices during the time period $T_{N 2}<t<T_{a m 2}$ (Table 1, see also Figure 3b). The characteristic 
frequencies and the observed peak flux are

$$
\begin{aligned}
\nu_{a, \mathrm{sd}}= & 1.7 \times 10^{12} \mathrm{~Hz} L_{0,49}^{-(7 p+10) / 2(p+4)} T_{\mathrm{sd}, 3}^{-(5 p+12) /(p+4)} \\
& \times M_{\mathrm{ej},-4}^{4(p+2) /(p+4)} \epsilon_{e}^{2(p-1) /(p+4)} \epsilon_{B,-1}^{(p+2) / 2(p+4)} \gamma_{4,4}^{2(p-2) /(p+4)} \\
\nu_{m, \mathrm{sd}}= & 1.2 \times 10^{12} \mathrm{~Hz} L_{0,49}^{-7 / 2} \epsilon_{B,-1}^{1 / 2} \epsilon_{e}^{2} \gamma_{4,4}^{2}\left(\frac{p-2}{p-1}\right)^{2} \\
\nu_{c, \mathrm{sd}}= & 1.9 \times 10^{16} \mathrm{~Hz} L_{0,49}^{13 / 2} T_{\mathrm{sd}, 3}^{9} M_{\mathrm{ej},-4}^{-8} \epsilon_{B,-1}^{-3 / 2} \\
F_{\nu, \text { max }, \mathrm{sd}}= & 3.5 \times 10^{5} \mathrm{mJy}_{0,49}^{-1 / 2} M_{\mathrm{ej},-4}^{2} \epsilon_{B,-1}^{1 / 2} \gamma_{4,4}^{-1} D_{27}^{-2} .
\end{aligned}
$$

For Case III the corresponding values are

$$
\begin{aligned}
T_{\mathrm{N} 1} & =2.1 \times 10^{-3} \text { days } L_{0,49}^{-1} M_{\mathrm{ej},-3} \\
T_{\mathrm{dec}} & =0.9 \text { days } L_{0,49}^{-7 / 3} T_{\mathrm{sd}, 3}^{-7 / 3} M_{\mathrm{ej},-3}^{8 / 3} n^{-1 / 3} \\
T_{\mathrm{N} 2} & =89.1 \text { days } L_{0,49}^{1 / 3} T_{s d, 3}^{1 / 3} n^{-1 / 3} \\
\gamma_{\mathrm{sd}} & =5.6 L_{0,49} T_{\mathrm{sd}, 3} M_{\mathrm{ej},-3}^{-1}+1,
\end{aligned}
$$

and

$$
\begin{aligned}
\nu_{a, \mathrm{sd}}= & 6.9 \times 10^{13} \mathrm{~Hz} L_{0,49}^{-(3 p+14) / 2(p+4)} T_{\mathrm{sd}, 3}^{-(3 p+14) /(p+4)} \\
& \times M_{\mathrm{ej},-3}^{2(p+5) /(p+4)} \epsilon_{B,-1}^{(p+2) / 2(p+4)} \gamma_{4,4}^{-2 /(p+4)} \\
\nu_{m, \mathrm{sd}}= & 2.3 \times 10^{15} \mathrm{~Hz} L_{0,49}^{-7 / 2} T_{\mathrm{sd}, 3}^{-5} M_{\mathrm{ej},-3}^{4} \gamma_{4,4}^{2} \epsilon_{e}^{2} \epsilon_{B,-1}^{1 / 2}\left(\frac{p-2}{p-1}\right)^{2} \\
\nu_{c, \mathrm{sd}}= & 1.8 \times 10^{10} \mathrm{~Hz} L_{0,49}^{-3 / 2} T_{\mathrm{sd}, 3}^{-3} M_{\mathrm{ej},-3}^{2} \epsilon_{B,-1}^{1 / 2} \\
F_{\nu, \max , \mathrm{sd}}= & 4.6 \times 10^{3} \mathrm{Jy} L_{0,49}^{-1 / 2} T_{\mathrm{sd}, 3}^{-2} M_{\mathrm{ej},-3}^{2} \epsilon_{B,-1}^{1 / 2} \gamma_{4,4}^{-1} D_{27}^{-2} .
\end{aligned}
$$

\section{Ionization Break Out}

\subsection{Opacity Estimate}

Our calculations above are based on the results of Kasen et al. (2013), who studied the opacity at optical/infrared wavelengths, in particular the bound-bound opacity. For UV radiation, we are interested in the radiation at early times, i.e. $t \lesssim 600 \mathrm{~s}$, because the ejecta will become transparent at later times. During such early times, the ejecta are hot enough to thermally ionize the lanthanides, e.g. Ce, to $\mathrm{Ce}^{3+}$. For simplicity, in the following calculations we assume that Ce is completely thermally ionized as $\mathrm{Ce}^{3+}$. As estimated below, 
the opacity at UV wavelengths is dominated by the bound-free (photoionization) transitions. We will therefore calculate the photoionization of $\mathrm{Ce}^{3+}$ by the $\mathrm{UV}$ radiation from $\mathrm{RS}$. The forth ionization potential of Ce is $\chi_{4}=36.72 \mathrm{eV}$ (Cox 2001). We first estimate the different opacities as follows.

The wavelength independent electron scattering opacity is (e.g., Kasen et al. 2013)

$$
\kappa_{\mathrm{es}}=\frac{\bar{x} \sigma_{T}}{\bar{A} m_{p}} \approx 0.4\left(\frac{\bar{x}}{\bar{A}}\right) \mathrm{cm}^{2} \mathrm{~g}^{-1},
$$

where $\bar{A}$ is the mean atomic weight of the ions, $\bar{x}$ the mean ionization fraction, $m_{p}$ the proton mass. After ultraviolet ionization, Ce is ionized to a level $\bar{x}=4$. With $\bar{A} \approx 140$ we find $\kappa_{\mathrm{es}} \approx 0.01 \mathrm{~cm}^{2} \mathrm{~g}^{-1}$, which is a factor of $\sim 20$ smaller than the usual value $\kappa_{\mathrm{es}}=0.2 \mathrm{~cm}^{2} \mathrm{~g}^{-1}$.

The free-free opacity can be found as (e.g., Rybicki \& Lightman 1979; Kasen et al. 2013)

$$
\kappa_{\mathrm{ff}}=0.15 \frac{\bar{x}^{3}}{\bar{A}^{2}} \rho_{-8} T_{5}^{-1 / 2} \lambda_{-5}^{3}\left(1-e^{-h c / \lambda k T}\right) \mathrm{cm}^{2} \mathrm{~g}^{-1},
$$

where the typical density of the ejecta at early times is $\rho \gtrsim 10^{-8} \mathrm{~g} \mathrm{~cm}^{-3}$ and temperature $T \gtrsim 2 \times 10^{5} \mathrm{~K} \simeq T_{\text {ion }}=\chi_{3} / k$, where $\chi_{3}=20.198 \mathrm{eV}$ is the third ionization potential of Ce, and $T_{\text {ion }}$ the corresponding temperature. For the values $\bar{x}$ and $\bar{A}$ mentioned above, we see that the free-free opacity at UV wavelengths is $\kappa_{\mathrm{ff}} \approx 5 \times 10^{-5} \mathrm{~cm}^{2} \mathrm{~g}^{-1}$, which is completely negligible. The free-free opacity at X-ray band is even smaller because of the dependence of opacity on wavelength $\lambda$.

The bound-bound opacities of the lanthanides are a function of both temperature and wavelength (Kasen et al. 2013). On the one hand, the line expansion opacity of cerium (Ce, $Z=58, f$-shell), for example, decreases to the red and sharply drops to zero for $\lambda \lesssim 1000 \AA$ (Figure 7 in Kasen et al. 2013). That is, the bound-bound opacity in the UV and X-ray bands is negligible at the temperature we are interested in, i.e. $T>T_{\text {ion. }}$ On the other hand, the Planck mean expansion opacity of neodymium $(\mathrm{Nd}, Z=60, f$-block, similar to Ce in shell structure) increases sharply with temperature when $T \lesssim 4000 \mathrm{~K}$ and then decreases with temperature (Figure 6 in Kasen et al. 2013). The reason is that, with the increase of temperature, more excited levels are populated. But if the gas becomes hot enough to ionize, the originally populated state leaves blank and the opacity declines. The boundbound opacity declines sharply when the temperature $T>15000 \mathrm{~K}$, i.e., $\kappa_{\mathrm{bb}} \lesssim 10^{-3} \mathrm{~cm}^{2} \mathrm{~g}^{-1}$ (Figure 9 of Kasen et al. 2013). For the temperature we are interested for UV radiation, i.e. $T>T_{\text {ion }}, \kappa_{\mathrm{bb}}$ becomes negligibly small.

The bound-free opacity at the threshold energy is given by (e.g., Kasen et al. 2013)

$$
\kappa_{\mathrm{bf}}=\frac{\sigma_{0}}{\bar{A} m_{p}} \frac{e^{-\Delta E / k T}}{Z(T)},
$$


where $Z(T)$ is the partition function and $\Delta E$ the excitation energy. The Boltzmann factor $e^{-\Delta E / k T}$ takes account of the fact that an atom has to be thermally excited so that a photon has enough energy to ionize the excited atom. The wavelength-dependent parameter $\sigma_{0}$ is not accurately constrained but can be approximated as the hydrogenic value $\sigma_{0} \approx 6 \times 10^{-18} \mathrm{~cm}^{2}$ at optical/UV wavelengths. For UV radiation, $\Delta E \approx 0$, unless the temperature $T \gg T_{\text {ion. }}$ The largest uncertainty for the calculation of $\kappa_{\mathrm{bf}}$ comes from the partition function $Z(T)$. Irwin (1981) tabulated $Z(T)$ for $\mathrm{Ce}^{+}$and $\mathrm{Ce}^{2+}$ in the temperature range $1000 \mathrm{~K}-16000 \mathrm{~K}$. The partition function increases slowly with temperature. It is also expected that the partition function of $\mathrm{Ce}^{3+}$ is smaller than $\mathrm{Ce}^{2+}$ at high temperature. Accordingly, we estimate $Z(T) \sim$ 1000 for $T \gtrsim T_{\text {ion. }}$. Taking the typical values, we find $\kappa_{\mathrm{bf}} \approx 25 e^{-\Delta E / k T} \mathrm{~cm}^{2} \mathrm{~g}^{-1}$ for $T \gtrsim T_{\text {ion }}$. We therefore conclude that for UV photons, bound-free opacity is dominant. For X-ray, $\Delta E=0$, but $\sigma_{0}$ takes a value several orders of magnitude smaller than it does at the optical wavelengths (Hakken \& Wolf 1987). Consequently, the bound-free opacity for X-ray is negligible and the opacity at X-ray is dominated by $\kappa_{\mathrm{es}}$.

Although the above reasoning is quite robust, but owing to the complex nature of opacities, it is worth of more scrutiny here. Figure 7 in Kasen et al. (2013) shows that the opacity of osmium (Os, $Z=76, d$-shell) is very high at UV wavelengths. But this high opacity is only for temperature $\sim 5000 \mathrm{~K}$. Figure 6 in Kasen et al. (2013) shows that the opacity of Fe (also $d$-shell in shell structure, therefore similar to Os) keeps constant in the temperature range $5000-14000 \mathrm{~K}$. But with a temperature high enough to ionize Os, the opacity will decline, as explained above. Consequently we can tentatively infer that the bound-bound opacity of Os at temperature $T_{\text {ion }}$ is smaller than the bound-free opacity evaluated in the previous paragraph. Another uncertainty comes from actinides $(90<Z<100)$. While the actinide series is generally of very low abundance, but because of the fact that line expansion opacities are expected to shift to shorter wavelengths for heavy elements such as actinides, the actinide series may make significant contribution to the opacity that exceeds the bound-free opacity estimated above. If the bound-bound opacity

contributed by Os and/or actinides is in excess of the bound-free opacity, we will not observe an UV ionization break-out.

\subsection{Ionization by ultraviolet radiation from $\mathrm{RS}$}

Before elaborating the calculation of ionization, we need evaluate the recombination factor, which can be calculated according to the bound-free opacity as (Rybicki \& Lightman 1979) 


$$
\alpha_{\mathrm{rec}}=4 \pi\left(\frac{m_{e}}{2 \pi k T}\right)^{3 / 2} \frac{\sigma_{0}}{m_{e}^{3} c^{2}} \frac{2 g_{n}}{g_{e} g_{+}} \frac{\chi^{3}}{Z(T)} e^{(\chi-\Delta E) / k T} \operatorname{Ei}\left(\frac{\chi}{k T}\right)
$$

where $g_{e}, g_{+}, g_{n}$ are the statistical weight factors of electron, $\mathrm{Ce}^{4+}$ and $\mathrm{Ce}^{3+}$, respectively, $m_{e}$ the electron mass, and $\operatorname{Ei}(x)$ is defined as

$$
\operatorname{Ei}(x)=\int_{x}^{\infty} \frac{e^{-t}}{t} d t .
$$

For $\mathrm{Ce}^{4+}$ we set $\chi=\chi_{4}$.

The width, $\Delta_{\text {ej }, i}^{\prime}$, of the ionized zone $\left(\mathrm{Ce}^{4+}\right)$ of the ejecta shell in the comoving frame evolves as (cf. Equation (9-8) of Harwit 2006)

$$
\frac{d \Delta_{\mathrm{ej}, i}^{\prime}}{d t^{\prime}}=\left(4 \pi r^{2} \xi n_{3+}\right)^{-1} \frac{d N_{i}}{d t^{\prime}}-\Delta_{\mathrm{ej}, i}^{\prime} n_{e} \alpha_{\mathrm{rec}}
$$

where

$$
n_{3+}=\frac{M_{\mathrm{ej}}}{4 \pi r^{2} \xi \Delta_{\mathrm{ej}}^{\prime} \bar{A} m_{p}}
$$

is the number density of $\mathrm{Ce}^{3+}$ before ionization by UV radiation and $d N_{i} / d t^{\prime}$ the injection rate of the ionizing photons from $\mathrm{RS}$. The electron density $n_{e}$ is $n_{e}=\bar{x} n_{4+}$, where $n_{4+}$ $\left(n_{4+}=n_{3+}\right)$ is the number density of $\mathrm{Ce}^{4+}$. By setting $n_{4+}=n_{3+}$, we neglect the thermal expansion of the photoionized zone due to the increase of pressure relative to the $\mathrm{Ce}^{3+}$ zone.

The UV photons from RS are scattered off free electrons before ionizing $\mathrm{Ce}^{3+}$ zone. The optical depth for the ionizing UV photons is

$$
\tau_{\mathrm{UV}}=\bar{A} m_{p} n_{3+} \kappa_{\mathrm{es}} \Delta_{\mathrm{ej}, i}^{\prime}
$$

The injection rate $d N_{i} / d t^{\prime}$ is therefore evaluated as

$$
\frac{d N_{i}}{d t^{\prime}}=4 \pi D_{L}^{2} \frac{F_{\nu^{\prime}}^{\prime} e^{-\tau_{\mathrm{UV}}}}{h \nu^{\prime}} \Delta \nu^{\prime}
$$

where $F_{\nu^{\prime}}^{\prime}=\mathcal{D}^{-3} F_{\nu}$ is the energy flux from RS in comoving frame, $h \nu^{\prime}=\chi_{4}$ is the energy of ionizing photons. The thermal broadening is given by

$$
\Delta \nu^{\prime}=\nu^{\prime} \sqrt{\frac{3 k T}{\bar{A} m_{p} c^{2}}}
$$

The thickness of the transition from $\mathrm{Ce}^{3+}$ zone to $\mathrm{Ce}^{4+}$ zone is of the order of mean free ionizing path $\delta=\left(n_{3+} \sigma_{\mathrm{bf}}\right)^{-1} \simeq 1.5 \times 10^{6} \mathrm{~cm} n_{3+, 14}^{-1}$, which is more than one order of magnitude smaller than the width of the ejecta $\Delta_{\text {ej }}^{\prime} \gtrsim 10^{7} \mathrm{~cm}$. 
The calculated UV light curves and ionization fraction $\left(\Delta_{\mathrm{ej}, i}^{\prime} / \Delta_{\mathrm{ej}}^{\prime}\right)$ are depicted in Figure 5. The observed UV flux is attenuated by a factor $e^{-\tau_{\mathrm{bf}}}$, where $\tau_{\mathrm{bf}}=\kappa_{\mathrm{bf}}\left(\Delta_{\mathrm{ej}}^{\prime}-\Delta_{\mathrm{ej}, i}^{\prime}\right) \bar{A} m_{p} n_{3+}$. Before the ejecta are completely photoionized, $\tau_{\text {bf }} \gtrsim 100$ so that effectively the UV flux can be observed only when the ejecta are completely photoionized, i.e. ionization break out. This is why we see an abrupt increase of UV flux when the ionization fraction equals 1 . As the ejecta expand, the ejecta temperature drops rapidly so that when $T=T_{\text {ion }}$ the thermally ionized $\mathrm{Ce}^{3+}$ begins to recombine. We see from Figure 5 that it is not long when the ejecta become transparent after the temperature drops below $T_{\text {ion. }}$. As a result, our treatment of the ultraviolet flux is not affected very much by the recombination.

\section{Discussion}

$R$-process material is of great importance astrophysically, the studies of which are unfortunately very difficult in laboratory. It is therefore particularly valuable if we can study their properties by observing radiation, obscured by a pure $r$-process material, at different wavelengths. To this aim, we propose in this paper to observe the RS emission obscured by the ejecta, which is believed to be a pure $r$-process material.

Figures 2-4 show that the ejecta shell is subrelativistic when it becomes transparent (at time $t_{\mathrm{x}, \text { thin }}$ ) to X-ray emission from RS. During this phase the radius of the ejecta shell, suitable for Case I, II, and III, is

$$
r=2.1 \times 10^{13} \mathrm{~cm} L_{0,47}^{1 / 2} M_{\mathrm{ej},-4}^{-1 / 2} t_{3}^{3 / 2}, \quad t<T_{N 1}
$$

from which $t_{\mathrm{x}, \text { thin }}$ can be estimated as

$$
t_{\mathrm{x}, \text { thin }}=7.1 \times 10^{2} \mathrm{~s} M_{\mathrm{ej},-4}^{2 / 3} L_{0,47}^{-1 / 3}
$$

upon substituting Equation (32). Inspection of Figures 2-4 indicates that $t_{\mathrm{x}, \text { thin }}$ is approximately the time when the X-ray flux peeks from the left edge of the light curve. This behavior is also true for optical flux. Note that this estimate for $t_{x, \text { thin }}$ is accurate enough in comparison with the numerically determined value.

The estimate for the time, $t_{\mathrm{opt}, \text { thin }}$, when the ejecta become optically thin for visible light can be done in a similar way as long as we are aware of the fact that at this time the ejecta shell is in relativistic regime so that the radius should be given by

$$
r=1.3 \times 10^{13} \mathrm{~cm} L_{0,47}^{2} M_{\mathrm{ej},-4}^{-2} t_{3}^{2}, \quad T_{N 1}<t<\min \left(T_{\mathrm{sd},} T_{\mathrm{dec}}\right)
$$

from which we get

$$
t_{\mathrm{opt}, \mathrm{thin}}=2.2 \times 10^{3} \mathrm{~s} \kappa_{\mathrm{bb}}^{1 / 6} M_{\mathrm{ej},-4}^{5 / 6} L_{0,47}^{-2 / 3} .
$$


Unfortunately, $t_{\mathrm{opt} \text {,thin }}$ depends on $\kappa_{\mathrm{bb}}$ very weakly so that only a loose constraint on $\kappa_{\mathrm{bb}}$ can be obtained. If, on the other hand, the ejecta are massive enough and/or the spindown luminosity of the central magnetar is moderate so that the ejecta shell is still in the subrelativistic regime when it becomes transparent for visible light, we have $t_{\mathrm{opt}, \text { thin }} \propto \kappa_{\mathrm{bb}}^{1 / 3}$ and $\kappa_{\mathrm{bb}}$ can be constrained more compactly.

To evaluate the dependence of $t_{\mathrm{UV}, \mathrm{BO}}$, the break-out time of UV radiation, on $\kappa_{\mathrm{bf}}$, we vary $\kappa_{\mathrm{bf}}$ by a factor $f$, i.e., $\kappa_{\mathrm{bf}}^{\prime}=f \kappa_{\mathrm{bf}}$. From Equations (34) and (35) we see that the factor $f$ contains the uncertainties in $\sigma_{0}$ and $Z(T)$. The uncertainty in $\sigma_{0}$ is a few, so is $Z(T)$. So we expect the uncertainty of $f$ is $\sim 10$. The solid lines from left to right in the insets of Figure 5 show the UV light curves with $f=0.1,1$, and 10 , respectively. This time lies

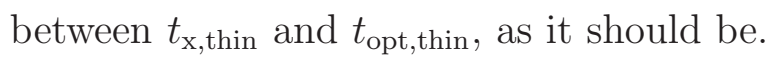

This model contains many free parameters to be constrained by fitting light curves. Wang \& Dai (2013b) demonstrated that by simultaneously fitting the optical and radio light curves, one can accurately constrain the spin-down luminosity $L_{\text {sd }}$, the spin-down time scale $T_{\text {sd }}$, the ejecta mass $M_{\mathrm{ej}}$, the density of ambient medium (region 1 ) $n$, the Lorentz factor of leptonized wind (region 4) $\gamma_{4}$, the electron power-law index of reverse shocked wind (region 3) $p^{\mathrm{RS}}$, the magnetic energy fraction $\epsilon_{B}$ and the redshift $z$ of the source (Table 2 of Wang \& Dai 2013b).

Wang \& Dai (2013b) also determined the launch time of the Poynting flux of PTF11agg. This is possible because the launch time affects the temporal decline index of the early optical light curve. To accurately determine the launch time of the Poynting flux by timing X-ray flux, Figures 2-4 indicate that we have to take observation in X-ray band following the merger no later than $\sim 100-700 \mathrm{~s}$. A similar observational strategy has to be employed to determine the ionization break-out of ultraviolet light (see Figure 5). Future gravitational observation of NSMs may also help determine the launch time of Poynting flux because this time roughly coincides with the merger time. Figure 5 shows that the ionization break-out time depends on $\kappa_{\mathrm{bf}}$ weakly. Thus by timing X-ray, UV, and visual light we can obtain weak constraints on $\kappa_{\mathrm{bb}}$ and $\kappa_{\mathrm{bf}}$.

\section{Conclusions}

In this paper, we have analyzed electromagnetic counterparts in various bands to the binary neutron star merger, and arrived at the following conclusions:

(i) By assuming that the Poynting flux of the central magnetar becomes lepton-dominated so that RS develops, broad-band EM signals, i.e. radio, optical, UV, and X-ray emis- 
sion, can be produced. The NSM ejecta can be accelerated to relativistic speed so that a forward shock can form. We therefore expect to observe EM signals first from RS and subsequently from FS.

(ii) In the study of the optical and radio radiation of PTF11agg, the simple dynamics, i.e., Equation (3), was adopted by Wang \& Dai (2013b). In this paper, the dynamics is determined by pressure balance and it is found that Equation (3) is an acceptable approximation.

(iii) In studying PTF11agg, Wang \& Dai (2013b) ignored the absorption of optical radiation by the ejecta. We find in this paper, by including the absorption effect, that by the time the first optical datum was taken, the ejecta become optically thin so that the treatment of Wang \& Dai (2013b) is justified. Wang \& Dai (2013b) also ignored the emission from the ejecta itself, which is verified in this paper (Figure 2) that the emission of the ejecta itself is negligible in all relevant bands.

(iv) Scattering off free electrons will obscure the early X-ray emission from RS. Optical emission from RS will be suppressed by bound-bound opacity until the ejecta become transparent at later times. UV radiation from RS, on the other hand, has the right energy to ionize the hot ejecta during its early expansion and ionization breakout should therefore be observed.

(v) Given the weak dependence of arrival times of EM signals at different wavelengths on different opacities, we conclude that the timing of X-ray, UV, and optical light is not an accurate method to observationally constrain the opacities of $r$-process material.

(vi) Recent papers by Gao et al. (2013) and Wang \& Dai (2013b) make an approximation that the merger ejecta is rapidly compressed into a thin expanding shell, similar to the one which forms at GRB afterglows. In this paper we estimate the time it takes for the flux to compress the ejecta to a thin shell and find that the time is negligible compared with the activity duration of the central magnetar. As a result, the models proposed by Gao et al. (2013) and Wang \& Dai (2013b) can be applied safely.

We are grateful to the referee for insightful comments and constructive suggestions, which significantly improve the presentation of this paper. This work is supported by the National Basic Research Program ("973" Program) of China under Grant No. 2014CB845800. L.J.W. and Z.G.D are also supported by the National Natural Science Foundation of China (grant No. 11033002), and Y.W.Y. by grant No. 11473008. 


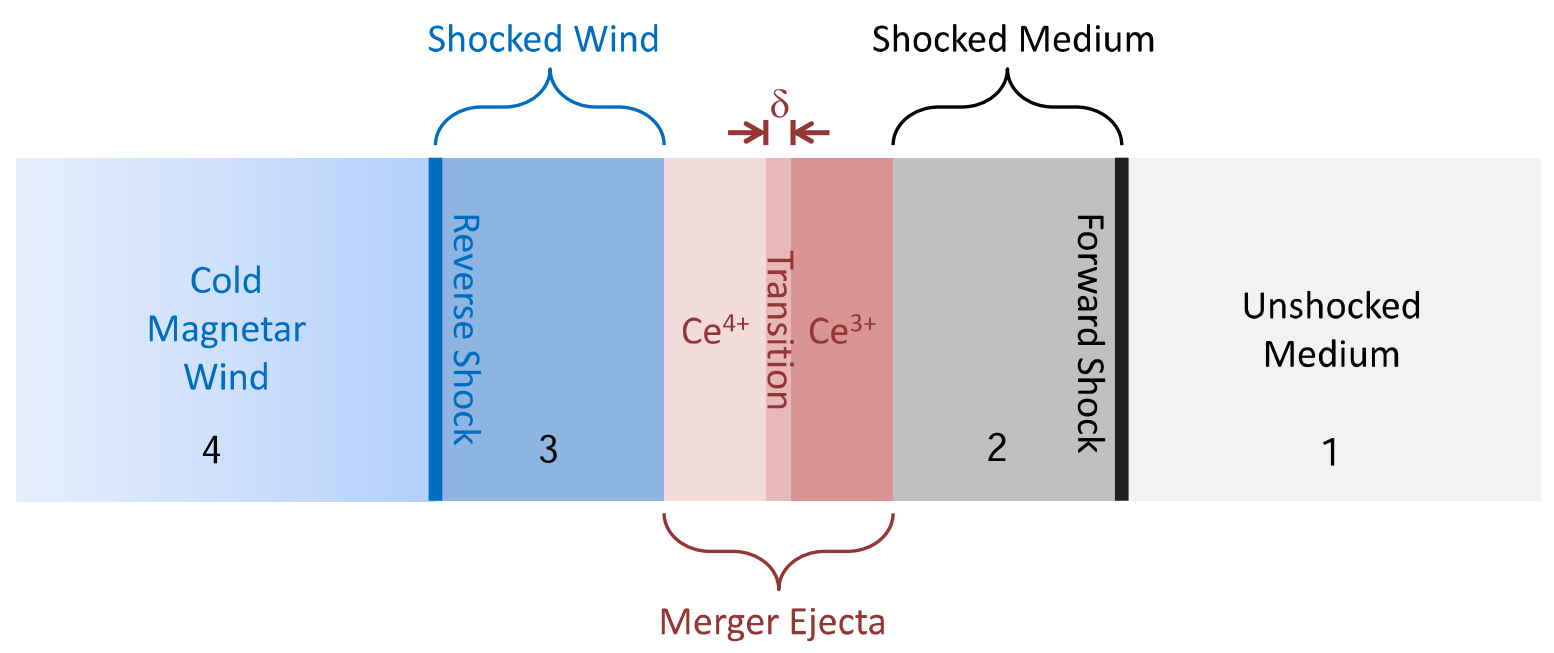

Fig. 1.- Schematic illustration of the physical picture described in this paper. The merger ejecta are catched up by the relativistic $e^{ \pm}$wind, driving the surrounding medium to form forward shock. The magnetar wind, on the other hand, is heated by the reverse shock. The ultraviolet radiation from the reverse shock ionizes the thermally ionized $\mathrm{Ce}^{3+}$ to $\mathrm{Ce}^{4+} . \delta$ is the transition width between $\mathrm{Ce}^{4+}$ zone and $\mathrm{Ce}^{3+}$ zone. When the ejecta temperature drops below $T_{\text {ion }}$, viz. the temperature to thermally ionize $\mathrm{Ce}$ to $\mathrm{Ce}^{3+}, \mathrm{Ce}^{3+}$ begins to recombine. Consequently, the ionization stage can only last for a limited time. The Arabic numbers indicate different regions: region 1 is the unshocked medium, region 2 is the shocked medium, region 3 is the shocked wind, region 4 is the unshocked cold wind. 

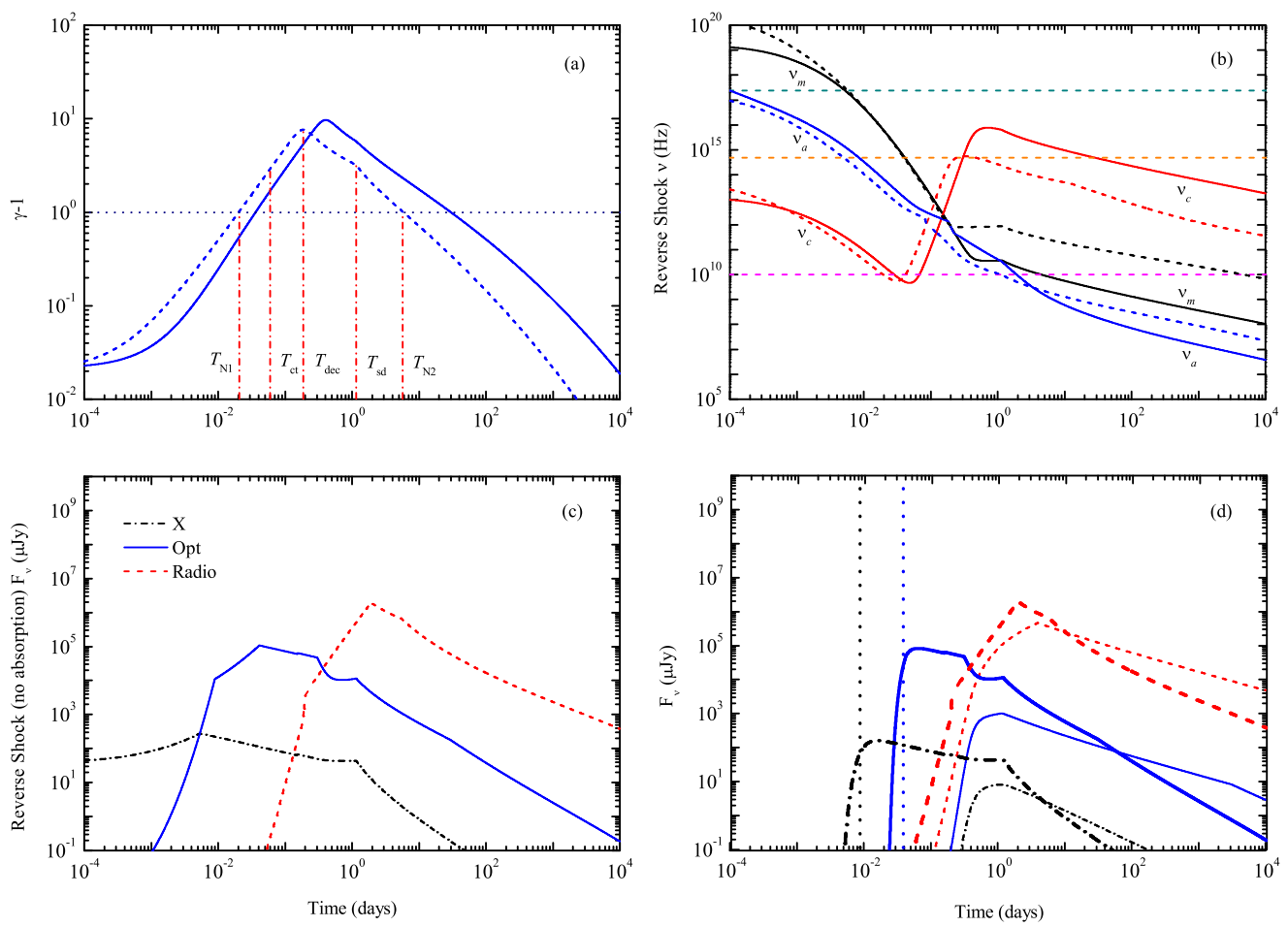

Fig. 2.- Calculation results for $L_{\mathrm{sd}, i}=10^{47} \mathrm{erg} \mathrm{s}^{-1}, T_{\mathrm{sd}}=10^{5} \mathrm{~s}$ and $M_{\mathrm{ej}}=10^{-4} M_{\odot}$. (a) The evolution of Lorentz factor. (b) The characteristic frequencies of RS emission. The three dashed lines mark the X-ray, optical $(R)$ and radio $(8 \mathrm{GHz})$ bands, respectively. (c) Light curves of RS without absorption by ejecta. (d) Light curves of RS with absorption by ejecta considered (thick) and of FS (thin). The two vertical dotted lines in panel (d) mark the times when the ejecta become transparent for X-ray and visual light respectively. The emission by the heated ejecta is negligible in this case, so we do not show them. Dashed lines in panels (a) and (b) are the results based on the dynamics expressed by Equation (3). In panel (a) $T_{\mathrm{N} 1}$ and $T_{\mathrm{N} 2}$ are the times when $\gamma-1=1$, i.e., the transition time between relativistic motion and Newtonian dynamics, $T_{\text {ct }}$ is the transition time for cooling Lorentz factor, see Wang \& Dai (2013b) for more explanation. In the calculations we set initial ejecta velocity $\beta_{\mathrm{ej}, 0}=0.2$. 

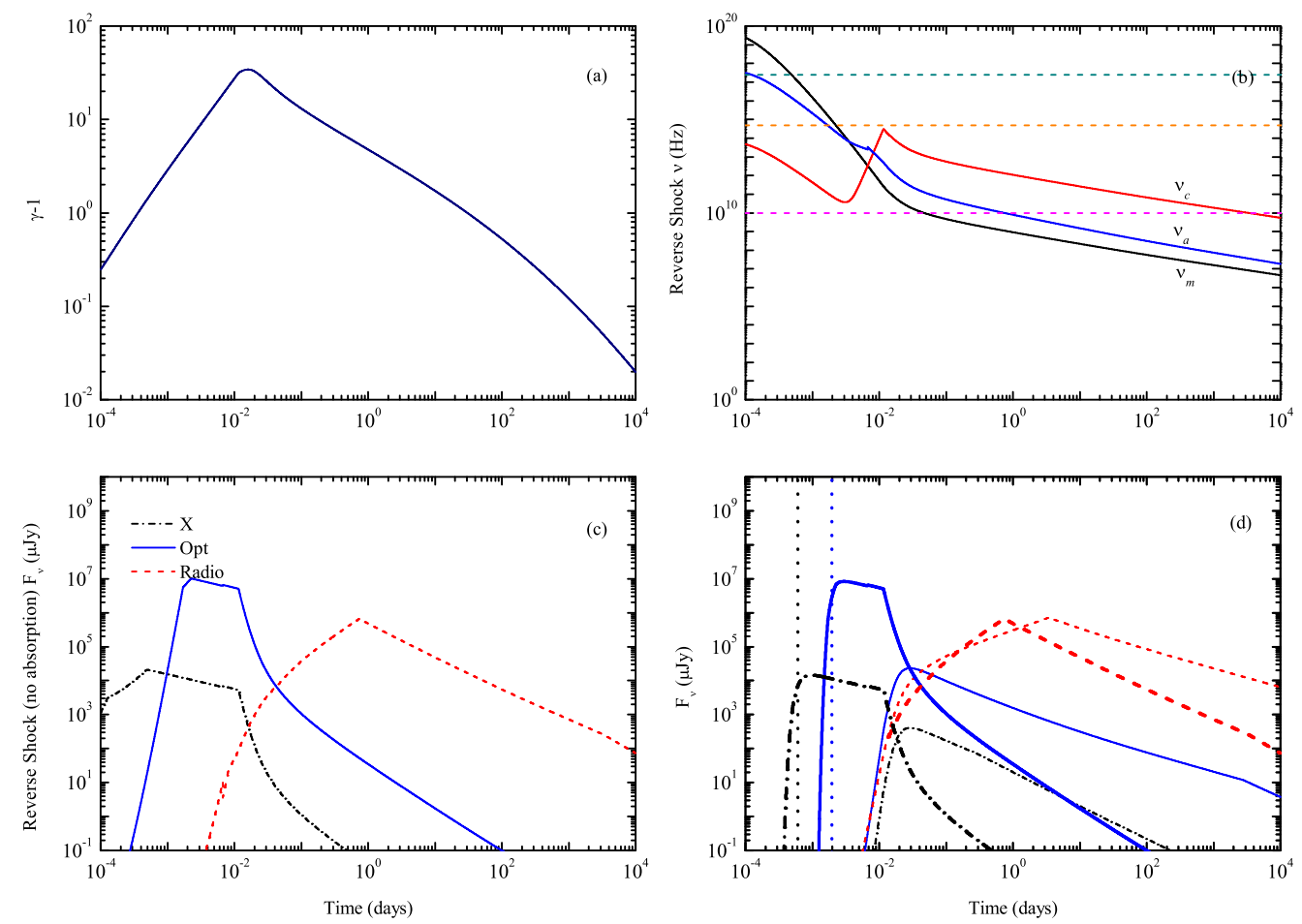

Fig. 3.- Calculation results for $L_{\mathrm{sd}, i}=10^{49} \mathrm{erg} \mathrm{s}^{-1}, T_{\mathrm{sd}}=10^{3} \mathrm{~s}, M_{\mathrm{ej}}=10^{-4} M_{\odot}$. The meanings of the panels are the same as in Figure 2. Ejecta emission is negligible. 

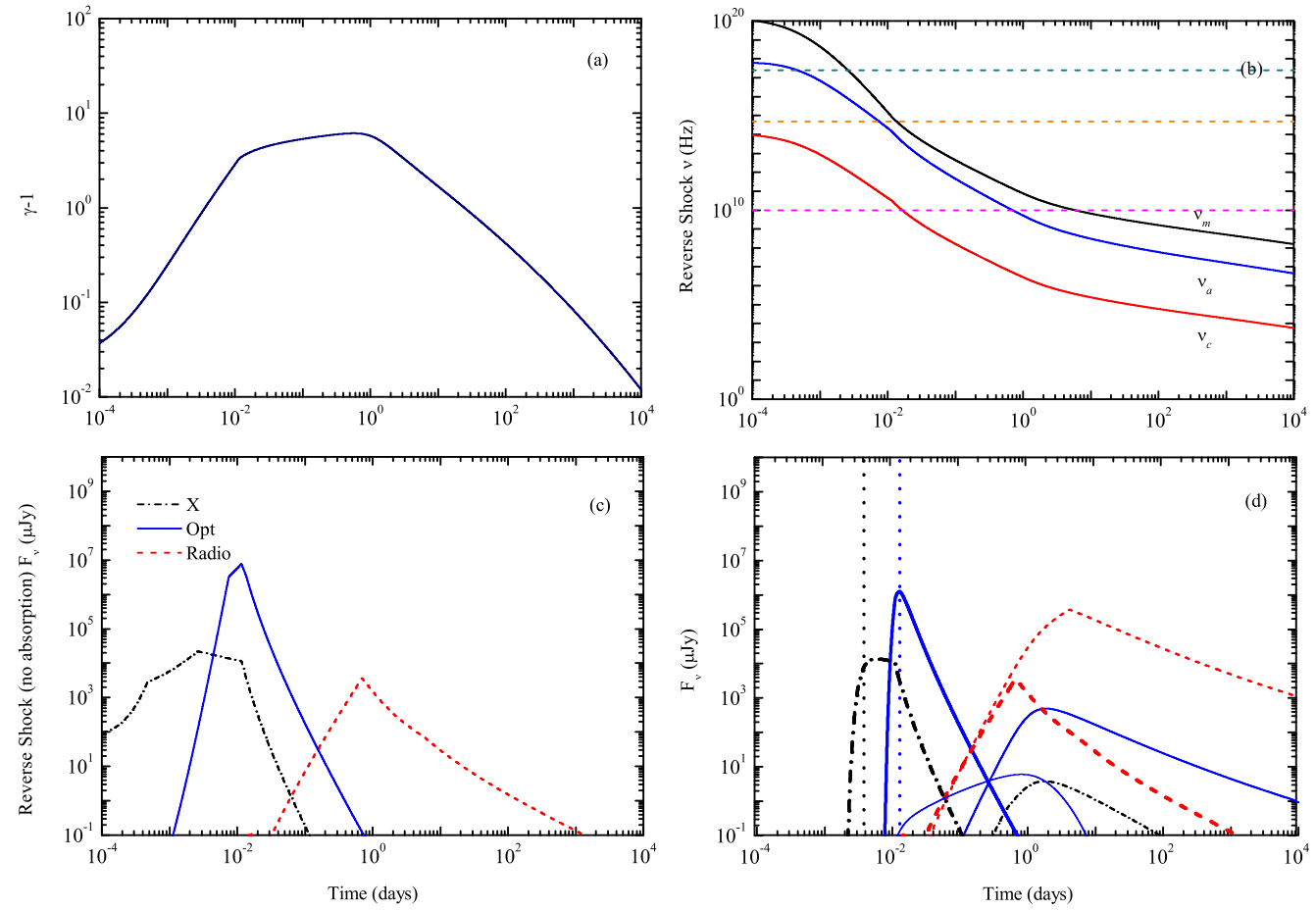

Fig. 4.- Calculation results for $L_{\mathrm{sd}, i}=10^{49} \mathrm{erg} \mathrm{s}^{-1}, T_{\mathrm{sd}}=10^{3} \mathrm{~s}, M_{\mathrm{ej}}=10^{-3} M_{\odot}$. The meanings of the panels are the same as in Figure 2. In panel $(\mathrm{d})$ the optical emission by the ejecta is shown as thin line. Ejecta emission in other bands is negligible. This is the only case discussed in this paper that the ejecta emission becomes appreciable in optical band. 


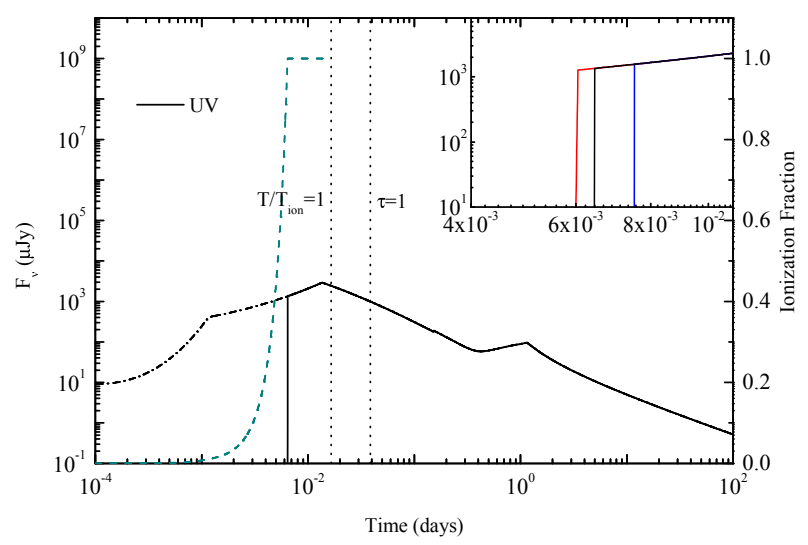

(a) $M_{\mathrm{ej}}=10^{-4} M_{\odot}, L_{\mathrm{sd}, i}=10^{47} \mathrm{erg} \mathrm{s}^{-1}$

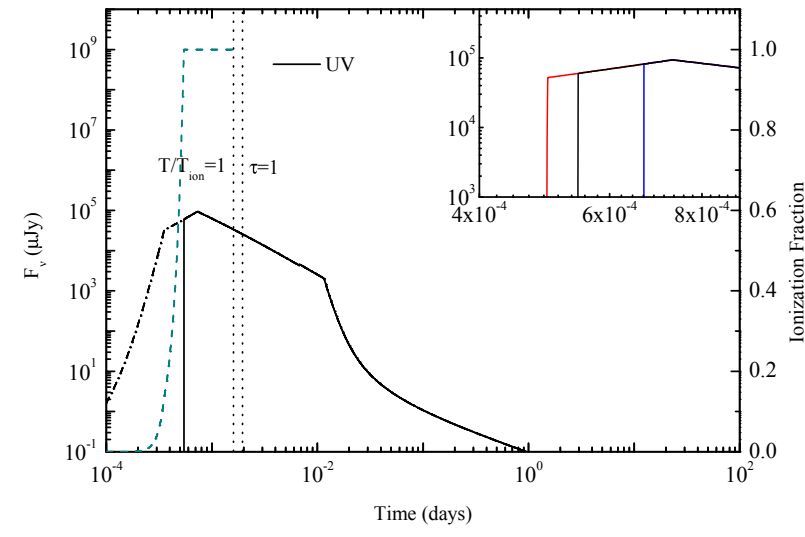

(b) $M_{\mathrm{ej}}=10^{-4} M_{\odot}, L_{\mathrm{sd}, i}=10^{49} \mathrm{erg} \mathrm{s}^{-1}$

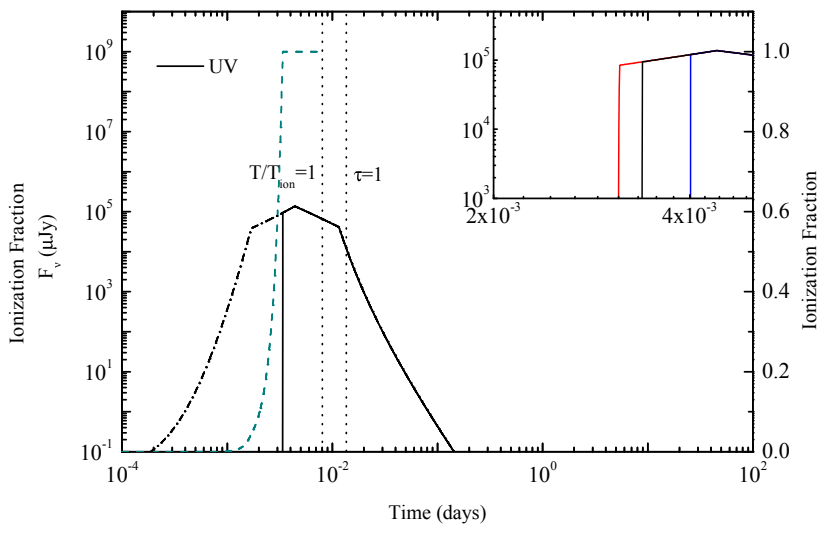

(c) $M_{\mathrm{ej}}=10^{-3} M_{\odot}, L_{\mathrm{sd}, i}=10^{49} \mathrm{erg} \mathrm{s}^{-1}$

Fig. 5.- Ultraviolet radiation from RS. The solid lines correspond to the observed ultraviolet flux leaked from the ionizing ultraviolet flux from RS (dash-dotted lines). The dashed lines are the ionization fraction of the ejecta. The vertical dotted lines indicate the times when $T / T_{\text {ion }}=1$ and $\tau=1$. The solid lines from left to right in the insets are the UV light curves by taking the bound-free opacity uncertainty factor $f=0.1,1$, and 10, respectively. 
Table 1: Analytical Temporal Scaling Indices of Various Parameters of the RS. The Analytical Results for Case I were Presented in Wang \& Dai (2013b).

\begin{tabular}{|c|c|c|c|c|c|c|}
\hline & $\gamma-1$ & $r$ & $\nu_{a}$ & $\nu_{m}$ & $\nu_{c}$ & $F_{\nu, \max }$ \\
\hline \multicolumn{7}{|c|}{ Case II: $L_{0} \sim 10^{49} \mathrm{erg} \mathrm{s}^{-1}, M_{\mathrm{ej}} \sim 10^{-4} M_{\odot}$} \\
\hline$t<T_{N 1}$ & 1 & $\frac{3}{2}$ & $-\frac{3 p+14}{2(p+4)}$ & $-\frac{3}{2}$ & $-\frac{3}{2}$ & $-\frac{1}{2}$ \\
\hline$T_{N 1}<t<T_{\mathrm{ct}}$ & 1 & 3 & $-\frac{3 p+14}{p+4}$ & -5 & -3 & -2 \\
\hline$T_{\mathrm{ct}}<t<T_{a c}$ & 1 & 3 & $-\frac{3 p+2}{p+4}$ & -5 & 9 & -2 \\
\hline$T_{a c}<t<T_{\mathrm{sd}}$ & 1 & 3 & $-\frac{5 p+12}{p+4}$ & -5 & 9 & -2 \\
\hline$T_{\mathrm{sd}}<t<T_{N 2}$ & $-\frac{3}{8}$ & $\frac{1}{4}$ & $-\frac{9 p+46}{16(p+4)}$ & $-\frac{9}{16}$ & $-\frac{17}{16}$ & $-\frac{9}{16}$ \\
\hline$T_{N 2}<t<T_{a m 2}$ & $-\frac{6}{5}$ & $\frac{2}{5}$ & $-\frac{3 p+14}{5(p+4)}$ & $-\frac{3}{5}$ & $-\frac{3}{5}$ & $-\frac{3}{5}$ \\
\hline$T_{a m 2}<t$ & $-\frac{6}{5}$ & $\frac{2}{5}$ & $-\frac{18}{25}$ & $-\frac{3}{5}$ & $-\frac{3}{5}$ & $-\frac{3}{5}$ \\
\hline \multicolumn{7}{|c|}{ Case III: $L_{0} \sim 10^{49} \mathrm{erg} \mathrm{s}^{-1}, M_{\mathrm{ej}} \sim 10^{-3} M_{\odot}$} \\
\hline$t<T_{N 1}$ & 1 & $\frac{3}{2}$ & $-\frac{3 p+14}{2(p+4)}$ & $-\frac{3}{2}$ & $-\frac{3}{2}$ & $-\frac{1}{2}$ \\
\hline$T_{N 1}<t<T_{\mathrm{sd}}$ & 1 & 3 & $-\frac{3 p+14}{p+4}$ & -5 & -3 & -2 \\
\hline$T_{\mathrm{sd}}<t<T_{\mathrm{dec}}$ & 0 & 1 & $-\frac{3 p+14}{2(p+4)}$ & $-\frac{3}{2}$ & $-\frac{3}{2}$ & $-\frac{3}{2}$ \\
\hline$T_{\mathrm{dec}}<t<T_{N 2}$ & $-\frac{3}{8}$ & $\frac{1}{4}$ & $-\frac{9 p+46}{16(p+4)}$ & $-\frac{9}{16}$ & $-\frac{9}{16}$ & $-\frac{9}{16}$ \\
\hline$T_{N 2}<t$ & $-\frac{6}{5}$ & $\frac{2}{5}$ & $-\frac{3 p+14}{5(p+4)}$ & $-\frac{3}{5}$ & $-\frac{3}{5}$ & $-\frac{3}{5}$ \\
\hline
\end{tabular}




\section{REFERENCES}

Abadie, J., Abbott, B. P., Abbott, R., et al. 2010, CQGra, 27, 173001

Atoyan, A. M. 1999, A\&A, 346, L49

Barnes, J. \& Kasen, D. 2013, ApJ, 775, 18

Barthelmy, S. D., Chincarini, G., Burrows, D. N., et al. 2005, Natur, 438, 994

Bartos, I., Brady, P., \& Márka, S. 2013, CQGra, 30, 123001

Bauswein, A., Goriely, S., \& Janka, H.-T. 2013, ApJ, 773, 78

Berger, E. 2007, ApJ, 670, 1254

Berger, E., Fong, W., \& Chornock, R. 2013, ApJL, 774, L23

Berger, E., Price, P. A., Cenko, S. B., et al. 2005, Natur, 438, 988

Cenko, S. B., Kulkarni, S. R., Horesh, A., Corsi, A., Fox, D. B. 2013, ApJ, 769, 130

Coroniti, F. V. 1990, ApJ, 349, 538

Cox, A. N. 2001, Allen's Astrophysical Quantities (Springer)

Dai, Z. G. 2004, ApJ, 606, 1000

Dai, Z. G. \& Liu, R.-Y. 2012, ApJ, 759, 58

Dai, Z. G., \& Lu, T. 1998a, A\&A, 333, L87

Dai, Z. G., \& Lu, T. 1998b, PhRvL, 81, 4301

Dai, Z. G., Wang, X. Y., Wu, X. F., \& Zhang, B. 2006, Sci, 311, 1127

Eichler, D., Livio, M., Piran, T., \& Schramm, D. N. 1989, Natur, 340, 126

Fan, Y. Z., \& Xu, D. 2006, MNRAS, 372, L19

Fan, Y.-Z, Yu, Y.-W., Xu, D., Jin, Z.-P., Wu, X.-F., Wei, D.-M. \& Zhang, B. 2013, ApJ, $779, \mathrm{~L} 25$

Faber, J. A., \& Rasio, F. A. 2012, LRR, 15, 8

Fong, W.-f., Berger, E., Metzger, B. D., et al. 2014, ApJ, 780, 118 
Fox, D. B., Frail, D. A., Price, P. A., et al. 2005, Natur, 437, 845

Gao, H., Ding, X., Wu, X. F., Zhang, B., \& Dai, Z. G. 2013, ApJ, 771, 86

Gao, W. H., \& Fan, Y. Z. 2006, ChJAA, 6, 513

Gehrels, N., Sarazin, C. L., O’Brien, P. T., et al. 2005, Natur, 437, 851

Giacomazzo, B., \& Perna, R. 2013, ApJL, 771, L26

Goriely, S., Bauswein, A., \& Janka, H.-T. 2011, ApJL, 738, L32

Gompertz, B. P., O’Brien, P. T., \& Wynn, G. A. 2014, MNRAS, 438, 240

Grossman, D., Korobkin, O., Rosswog, S., \& Piran, T. 2014, MNRAS, 439, 757

Hakken, H., \& Wolf, H. C. 1987, Atomic and Quantum Physics, Springer-Verlag

Harwit, M. 2006, Astrophysical Concepts, Springer

Hotokezaka, K., Kiuchi, K., Kyutoku, K., et al. 2013, PhRvD, 87, 024001

Huang, Y. F., Dai, Z. G., \& Lu, T. 1999, MNRAS, 309, 513

Irwin, A. W. 1981, ApJS, 45, 621

Jin, Z. P., Xu, D., Fan, Y. Z., et al. 2013, ApJL, 775, L19

Kasen, D., Badnell, N. R., \& Barnes, J. 2013, ApJ, 774, 25

Kawanaka, N., Mineshige, S., \& Piran, T. 2013, ApJL, 777, L15

Kohri, K., \& Mineshige, S. 2002, ApJ, 577, 311

Korobkin, O., Rosswog, S., Arcones, A., \& Winteler, C. 2012, MNRAS, 426, 1940

Kulkarni, S. R. 2005, arXiv:astro-ph/0510256

Landau, L. D. \& Lifshitz, E. M. 1987, Fluid Mechanics, Pergamon Press

Li, L.-X., \& Paczyński, B. 1998, ApJL, 507, L59

Metzger, B. D., Arcones, A., Quataert, E., Martinez-Pinedo, G. 2010a, MNRAS, 402, 2771

Metzger, B. D., \& Berger, E. 2012, ApJ, 746, 48

Metzger, B. D., \& Bower, G. C. 2014, MNRAS, 437, 1821 
Metzger, B. D., Martínez-Pinedo, G., Darbha, S., et al. 2010b, MNRAS, 406, 2650

Metzger, B. D., \& Piro, A. L. 2014, MNRAS, 439, 3916

Michel, F. C. 1994, ApJ, 431, 397

Nakar, E., \& Piran, T. 2011, Natur, 478, 82

Narayan, R., Piran, T., \& Kumar, P. 2001, ApJ, 557, 949

Paczyński, B. 1986, ApJL, 308, L43

Palenzuela, C., Lehner, L., Ponce, M., et al. 2013, PhRvL, 111, 061105

Piran, T., Nakar, E., \& Rosswog, S. 2013, MNRAS, 430, 2121

Popham, R., Woosley, S. E., \& Fryer, C. 1999, ApJ, 518, 356

Rezzolla, L., Baiotti, L., Giacomazzo, B., et al. 2010, CQGra, 27, 114105

Rezzolla, L., Giacomazzo, B., Baiotti, L., et al. 2011, ApJL, 732, L6

Roberts, L. F., Kasen, D., Lee, W. H., \& Ramirez-Ruiz, E. 2011, ApJL, 736, L21

Rosswog, S. 2005, ApJ, 634, 1202

Rosswog, S., Korobkin, O., Arcones, A., Thielemann, F.-K., \& Piran, T. 2014, MNRAS, 439,744

Rosswog, S., Piran, T., \& Nakar, E. 2013, MNRAS, 430, 2585

Rowlinson, A., O'Brien, P. T., Metzger, B. D., et al. 2013, MNRAS, 430, 1061

Rowlinson, A., O'Brien, P. T., Tanvir, N. R., et al. 2010, MNRAS, 409, 531

Rybicki, G. B., \& Lightman, A. P. 1979, Radiative Processes in Astrophysics (New York: Wiley-Interscience)

Soderberg, A. M., Berger, E., Kasliwal, M., et al. 2006, ApJ, 650, 261

Takami, H., Nozawa, T., \& Ioka, K. 2014, ApJ, 789, L6

Tanaka, M., \& Hotokezaka, K. 2013, ApJ, 775, 113

Tanvir, N. R., Levan, A. J., Fruchter, A. S., et al. 2013, Natur, 500, 547

Wang, F. Y., \& Dai, Z. G. 2013a, NatPh, 9, 465 
Wang, L. J., \& Dai, Z. G. 2013b, ApJL, 774, L33

Wu X.-F., Gao H., Ding X., Zhang B., Dai Z.-G., Wei J.-Y., 2014, ApJL, 781, L10

Xue, L., Liu, T., Gu, W.-M., \& Lu, J.-F. 2013, ApJS, 207, 23

Yu, Y. W., \& Dai, Z. G. 2007, A\&A, 470, 119

Yu, Y. W., Zhang, B., \& Gao, H. 2013, ApJL, 776, L40

Zhang, B. 2013, ApJL, 763, L22

Zhang, B., \& Mészáros, P., 2001, ApJ, 552, L35 